\title{
Ensuring Inclusivity in the 2023 Planetary Science and Astrobiology Decadal Survey
}

\section{Planetary Science 2023 Decadal Survey White paper on the State of the Profession}

Authors: Julie A. Rathbun, Planetary Science Institute (rathbun@psi.edu; she/her/hers) Christina Richey, Jet Propulsion Laboratory, California Institute of Technology Barbara A. Cohen, NASA Goddard Space Flight Center Jennifer L. Piatek, Central Connecticut State University James H. Roberts, Johns Hopkins Applied Physics Laboratory Ingrid J. Daubar, Brown University Serina Diniega, Jet Propulsion Laboratory, California Institute of Technology Sarah Hörst, Johns Hopkins University Aparna Venkatesan, University of San Francisco

\section{Co-signers:}

This whute paper is submitted as part of a collaborative effort organized by the Equity, Diversity, and Inclusion Working Group (EDIWG), a cross Assessment Group (AG) committee.

As of September 15th, this paper has be co-signed by over 50 people, whose names and affiliations can be found at https://docs.google.com/spreadsheets/d/12Q5BA9Lf3aZJvBqYRSl8ffx08fvWvIbRzdvJkAh $\underline{\text { S4as/edit?usp=sharing }}$

We continue to accept new co-signatures. If interested, please fill out this form: https://docs.google.com/forms/d/e/1FAIpQLSc2pPfhekLU2-yHlfoOv4TTRQNyyYtB1bMZ3 UavacVjWfBjyQ/viewform?usp=sf_link

Acknowledgements: A portion of the work described was carried out at the Jet Propulsion Laboratory, California Institute of Technology, under a contract with the National Aeronautics and Space Administration (80NM0018D0004). 
Inclusivity is a necessary goal of any process that aims to represent the consensus of a large group as it is "the practice or policy of including people who might otherwise be excluded or marginalized, such as those who have physical or mental disabilities and members of minority groups." Inclusivity is a necessary and achievable goal for both the Decadal Survey process and product, and for the NASA Planetary Science Enterprise guided by the Decadal Survey report.

In planetary science, there are many groups that have historically been excluded, including scientists of color and white women (Rivera-Valentín, et al., 2020). These are also the same groups that have been disproportionately affected by the current global pandemic and civil unrest. Before the murder of George Floyd by Minneapolis police triggered large-scale protests in the US and beyond, the Women in Planetary Science blog had a post titled "What are the impacts of performing a Decadal Survey during a global pandemic?"1 This post outlined how the pandemic was affecting scientists and how the general productivity of women, in particular, has been much lower likely due to the traditional uneven load of homekeeping. It further pointed out how racial and ethnic minority communities have been disproportionately affected by COVID-19 suggesting that members of these communities, even if not directly affected by the virus, are likely experiencing higher levels of stress and difficulty. Extended shelter-in-place orders during the pandemic have increased the productivity of only a segment of our profession while greatly exacerbating environmental/accessibility factors impacting research output and learning for many vulnerable populations. There is an already documented drop in journal articles led by women, and by mothers facing the "maternal wall" when trying to work from home while managing caregiving and remote learning environments for their child(ren). ${ }^{2}$

The civil unrest that has occurred over the last few months may have been triggered by the murder of George Flloyd, but violence against Black Americans has been occurring for more than 400 years and increasing in recent years ${ }^{3}$. Given the current state, it may be unrealistic to expect our Black colleagues to write white papers in addition to doing their usual work and all of the unpaid time, energy, and emotional labor that we regularly demand of them ${ }^{4}$. Black scientists are THE most underrepresented group in planetary science (Rathbun et al., 2020; Rivera-Valentín, et al., 2020). Furthermore, as we are editing this paper, several national disasters (hurricanes, fires) are affecting the US and these also have a disproportionate effect on marginalized groups.

The demographic data clearly demonstrates that Planetary Science has a problem with diversity, inclusion, and equity. If we want the next planetary decadal survey to help with that problem, or at least not exacerbate it, we need to hear from the voices that are most

1

https://womeninplanetaryscience.wordpress.com/2020/05/14/what-are-the-impacts-of-performing-a-deca dal-survey-during-a-global-pandemic/\#more-5732

${ }^{2}$ http://womeninastronomy.blogspot.com/2020/07/the-fallout-from-covid-19-on-astronomys.html

${ }^{3}$ https://www.theroot.com/a-timeline-of-events-that-led-to-the-2020-fed-up-rising-1843780800

${ }^{4}$ See, for example https://www.naiabutlercraig.com/post/sorry-i-can-t-just-focus-on-the-science and https://www.nature.com/articles/d41586-020-01883-8 
often missing, the very same voices that are least able to contribute at present owing to numerous crises.

\section{Problem: How can we ensure that the voices of the most marginalized in Planetary science are represented in the current Decadal Survey Process?}

NASA has an explicit goal to support a sustainable and diverse workforce, as stated in all high-level NASA strategic documents. The NASA Science Vision (Science 2020-2024: A Vision for Scientific Excellence) identified "Increase the diversity of thought and backgrounds represented across the entire SMD portfolio through a more inclusive and accessible environment" as one of its top priorities (Strategy 4.1). The 2018 NASA Strategic Plan identified "Cultivate a diverse and innovative workforce with the right balance of skills and experience to provide an inclusive work environment in which employees that possess varying perspectives, education levels, life experiences, and backgrounds can work together and remain fully engaged in our mission" as one of its top objectives (Strategic Objective 4.4). It could be argued that diversity, equity, and inclusion are more central to NASA's goals than almost any one particular planetary science investigation. For example, "diversity" is mentioned 7 times in Science Vision and 10 times in the Strategic Plan. In contrast, Europa is mentioned once in the Science Vision and not mentioned within the Strategic Plan.

Additionally, the statement of task for the decadal states that "The report should provide a clear exposition of ... The state of the profession including issues of diversity, inclusion, equity, and accessibility, the creation of safe workspaces, and recommended policies and practices to improve the state of the profession. Where possible, provide specific, actionable and practical recommendations to the agencies and community to address these areas." While this is the third decadal survey covering planetary science, it is the first time the statement of task includes a direction to consider diversity, equity, inclusion, and accessibility (DEIA). As such, the planetary science community has no experience in how to accomplish this goal, so we should learn from others. The last two Astronomy decadal surveys, most recently Astro2020, have been asked to comment on the "state of the profession" and they have learned in the process. The planetary science and astrobiology decadal survey should learn lessons from how Astro 2020 has addressed this goal.

Problem: How can we ensure that DEIA and the state of the profession are given the consideration they require in order to make recommendations that will improve the inclusivity of the Planetary Science and Astrobiology communities?

Here, we make recommendations to address the problems stated above. Note that in these recommendations, we often use the phrase underrepresented racial and ethnic groups. In planetary science, this means African American, Latin American, Pacific Islander, and Native American/indigenous scientists (Rivera-Valentín, et al., 2020). We also refer the reader to a white paper submitted to Astro2020 by D. Norman et al., titled "Providing a 
Timely Review of Input Demographics to Advisory Committees" which includes background on why a diverse committee is important.

Recommendation \#1: The Decadal Survey co-chairs should delay the white paper deadline. We include this recommendation as it was written before the white paper deadlines were subsequently delayed. We appreciate that the co-chairs were able to delay and stagger the deadlines for white paper input. Not only did this give more time for the papers to be written, but staggering the deadlines enabled authors to give time to state of the profession papers without sacrificing the time they needed to dedicate to science white papers. We also note, though, that while this helped some people, a few weeks to months extension did not help all people grappling with the sudden and large life/work challenges arising from the pandemic, natural disasters and other crises of the past few months .

Recommendation \#2: The Decadal Survey should have a panel that focuses specifically on the state of the profession and this panel should be charged with the need to make planetary science more diverse and inclusive. This panel should have more representation on the steering committee than science panels (see rec 3.4). If Academy membership does not reflect the diversity needs of the community, then membership requirements should be revisited, relaxed or waived. Note that it is our understanding that the state of the profession panel in the latest Astronomy Decadal Survey was able to relax some rules as they had 2 members from the same institution. Since the Astronomy community has had a "State of the Profession" panel on their last 2 decadal surveys, we should learn from their experiences.

Recommendation \#2.1: This state of the profession panel must "provide specific, actionable and practical recommendations to the agencies and community" as required by the statement of task. This is in contrast to findings, which, while necessary, are not sufficient. For more details, see Rigby et et al. (2019).

Recommendation \#2.2: Any report of this panel that does not make it into the final survey report should be publicly released.

Recommendation \#2.3: Involve social scientists in the "state of the profession panel" as panelists. These should be individuals with expertise in organizational management and DEIA.

Recommendation \#3: The Decadal Survey co-chairs need to ensure that voices of underrepresented groups, particularly African-American, Latin American and indigenous scientists, are represented in the final survey report. There are several ways to accomplish this goal. Using multiple methods ensures the broadest possible participation and is necessary in case any one of the methods alone become difficult.

Recommendation \#3.1: Make sure the committees, particularly the steering committee, included members of different demographic groups. We applaud the selection of Robin Canup as a co-chair of the steering committee, particularly as she is the 
first woman to lead a planetary decadal survey. However, planetary scientists often make the mistake of considering only gender when considering demographic diversity. Racial and ethnic minority groups are the most underrepresented in planetary science, and they must be included on committees. Furthermore, scientists with disabilities and LGBTQ+ scientists also need to be included. It is not enough to just include a few members of underrepresented groups. Their voices and input as a community also need to be heard and fully considered, and we must also keep in mind the challenges and burdens faced by those with intersectional identities. Studies show that members of underrepresented groups have trouble being heard during deliberations. The next two recommendations are made to ensure that doesn't occur.

Recommendation \#3.2: Ensure that the voices of all panel members are heard, particularly those that are members of underrepresented groups. To accomplish this, each Decadal Survey panel should appoint 2 members as the "equity facilitators", similar to "diversity facilitator" positions on some faculty search committees. Their role, which should be outlined in panel rules, would be to amplify voices of members of underrepresented groups and bring up DEIA issues when applicable to panel conversations. These individuals should be familiar with DEIA issues and receive additional training in DEIA issues (see recommendation 4.1 below). ${ }^{5}$

Recommendation \#3.3: The Decadal Survey Steering committee (and other committees) should set aside time in their schedules for presentations (on any topic, including science, missions, and state of the profession) by scientists who have not offered other input (such as lead authorship on a white paper). Presentations by members of underrepresented groups, particularly BIPOC scientists should be prioritized. While delaying the white paper deadlines enabled more scientists to participate in the process, the current pandemic and civil unrest disproportionately affect members of these groups. An additional 10 days on the science white paper deadline may not have been enough time to enable members of these groups to contribute to white papers as the events were still on-going and this change was late in the writing process of many white papers. The survey committees should ensure there are open and well-advertised (in advance) opportunities for these presentations, and responses to those opportunities by BIPOC/underrepresented groups should be prioritized.

Recommendation \#3.4: Put priority on demographic diversity over other diversity considerations. While it is laudable to aim for diversity in institution type, career stage, and scientific expertise, it is difficult to achieve diversity along so many axes within any small group. For example, scientists who are members of underrepresented groups are

\footnotetext{
${ }^{5}$ Because the committees can not grow to be too large, these committee members will also be scientists with their own area of scientific expertise. As such, they should be given the space to bring their science expertise to the table, along with their EDIA expertise. This means they may sometimes need to do twice the mental work to keep both areas of expertise ready and available - so giving them space, when appropriate, to bring their science expertise in (perhaps by having another person explicitly watching/thinking with an EDIA-focused perspective when a topic is in their science wheelhouse, or giving them time to respond over email rather than doing everything in a meeting, in real time) would help.
} 
more often earlier in their career than members of majority groups. And, they are disproportionately represented in certain institution types. As in rec. \#2, if Academy membership does not reflect the diversity needs of the community, then membership requirements should be revisited, relaxed or waived for committees in general and not just any "State of the Profession" panel.

Recommendation \#4: Advisory committees that collect community input, (e.g., the Decadal Survey review committee, PAC, and CAPS), also should collect, compile and review input demographic data before finalizing reports (e.g., the final Astro2020 Survey Report). A summary of these data should be released alongside the final survey report. For more details, see Norman et et al. (2019). Example questions for a demographic survey of committee participants is given in the appendix. Note that we asked the NAS to collect demographic data at the point of receipt of white papers, which they explicitly declined to do. This is strongly at odds with best practices and should be implemented before any other Planetary report conducted by the NAS.

\section{Recommendation \#5: Members of the Decadal Survey committees should understand and, as NASA states in their ROSES AO, "recognize and support the benefits of having diverse and inclusive scientific, engineering, and technology communities".}

Recommendation \#5.1: All Decadal Survey committee members should undergo
implicit bias and racial sensitivity training as early in the process as possible. In order to truly understand the benefits of inclusive groups, the committee members should receive appropriate training. This should include training on how to amplify voices of the most marginalized members of the community. The best timing for this training would be before beginning deliberations, but, at the latest, should occur before writing the report.

Recommendation \#5.2: If panels receive input (such as white papers) from groups that are not diverse, the scientific value and completeness of that input should be more deeply interrogated by the panel than input from diverse groups.

Recommendation \#5.3: The Decadal Survey Steering committee should set aside time in their schedule for presentations by the EDI working group that was formed by the AGs (or other established EDIA groups within the Planetary Science and Astrobiology communities, such as the DPS PCCS). When time is short and people are stressed, EDI goals are typically among the first to be dropped. Giving the leaders of the EDI working group the opportunity to present would help fill in the gap, particularly if there is no "State of the Profession" panel explicitly created for the current planetary sciences decadal survey.

\section{References}

Rivera-Valentín, E.G., et al. (2020) Who is Missing in Planetary Science?: A demographic study of the planetary science workforce?. White paper to the Planetary Science and Astrobiology Decadal Survey 2023-2032. 
Hendrix, A. et al. (2020) INSERT TITLE HERE. White paper to the Planetary Science and Astrobiology Decadal Survey 2023-2032.

Norman, D. et al. (2019) Providing a Timely Review of Input Demographics to Advisory Committees. White paper to the Astronomy and Astrophysics Decadal Survey 2020.

Rigby, J. et al. (2019) Astro2020 must issue actionable recommendations regarding diversity, inclusion, and harassment. White paper to the Astronomy and Astrophysics Decadal Survey 2020

\section{Appendix}

This appendix gives suggestions for the wording of demographic survey questions. The structure of these questions has been vetted and used by Susan White, interim director of the AIP's Statistical Research Center. The questions match terminology used in the DPS Workforce Survey. See Hendrix et al., (2020) and https://dps.aas.org/reports for additional details and the survey results.

\section{Sample Demographic Survey Questions:}

- Check here if you have already submitted information for this survey. Put at beginning of survey and no need to answer any questions if already submitted survey info.

- Which of the following employment sectors best describes your primary employer? University, research institute, Two-year college, Museum or planetarium, Telescope or observatory, NASA lab, Other federal agency, Self-employed, Other

- What is the highest degree you have received? Four-year college or university degree/Bachelor's degree, Some postgraduate or professional schooling, no postgraduate degree (e.g. some graduate school), Master's degree, Doctorate degree, Other professional degree

- In what year did you earn your highest degree?

- What is your current employment status at your primary employer? Tenured or tenured-equivalent, Tenure-track or equivalent, Hard Money/permanent/regular employee, Soft Money, Limited Term/postdoctoral appointment Freelance, Other

- What are your primary planetary research interests? Geology or Geomorphology, Geophysics, Geochemistry, Atmospheric science, Dynamics or Celestial mechanics, Magnetospheric science, Astrobiology, Solar System Origins, Extrasolar planets, Other

- What is your gender identity? Woman, Man, Another identity - (please specify if you wish), Prefer not to answer

- Do you identify as transgender? Yes No, Prefer not to answer

- Do you identify as: Heterosexual, Gay or Lesbian, Bisexual, Other (please specify if you wish), Prefer not to answer 
- Which of the following racial/ethnic designations best describes you? Please select all that apply. Hispanic/Latinx, American Indian or Alaska Native, Asian or Asian American, Black or African American, Native Hawaiian or Other Pacific Islander, White, Additional (please specify if you wish), Prefer not to answer

- Please indicate which of the following apply to you. Please check all that apply. I am deaf or hard-of-hearing, I have difficulty seeing even when wearing glasses, I have serious difficulty standing, walking, or climbing stairs, I have a cognitive or learning disability, I have a mental illness, I am neuroatypical, I have an autoimmune or pain disorder, or other chronic condition, I have disabling allerg, None of the above, Prefer not to answer

- Which of the following accessibility aids do you use? Please check all that apply. Sign language, American or other, Hearing aids, headphones, and other audio devices, Closed-captioning, Screen readers, Braille, Speech transcription, Mobility aids, Note takers, Quiet spaces, Dietary accommodation, Environmental adjustments - For example lighting, air, noise, etc., Service animal, Other accessibility aid (please specify), None of the above, Prefer not to respond 